\title{
Genetically-modified MAGE-A3-expressing MG1 Maraba Virus Vaccine
}

National Cancer Institute

\section{Source}

National Cancer Institute. Genetically-modified MAGE-A3-expressing MG1 Maraba Virus

Vaccine. NCI Thesaurus. Code C118973.

A vaccine consisting of the attenuated, genetically-modified, oncolytic form of the Maraba virus, MG1, which has been eng ineered to express a gene encoding the cancer testis antigen melanoma antigen family A3 (MAGE-A3), with potential antineoplastic activity. Upon administration of genetically-modified MAGE-A3-expressing MG1 Maraba virus vaccine, the attenuated Maraba virus selectively and rapidly replicates in cancer cells; however, it is unable to replicate in normal, healthy cells. This induces a selective Maraba virus-mediated cytotoxicity in those cancer cells, and leads to cancer cell lysis. In addition, the expression of MAGE-A3 further stimulates the host immune system to mount a cytotoxic T-lymphocyte ( $C T L$ ) response against tumor cells expressing MAGE-A3. The tumor-associated antigen MAGE-A3 is overexpressed by a variety of cancer cell types. The attenuated virus is a double mutant strain with single amino acid substitutions occurring in both G protein (Q242R) and M protein (L123W). 